\title{
Application of Sinc-Galerkin Method for Solving Space-Fractional Boundary Value Problems
}

\author{
Sertan Alkan ${ }^{1}$ and Aydin Secer ${ }^{2}$ \\ ${ }^{1}$ Department of Management Information Systems, Bartin University, 74100 Bartin, Turkey \\ ${ }^{2}$ Department of Mathematical Engineering, Yildiz Technical University, 34220 Istanbul, Turkey \\ Correspondence should be addressed to Aydin Secer; asecer@yildiz.edu.tr
}

Received 10 June 2014; Accepted 13 July 2014

Academic Editor: Abdon Atangana

Copyright (c) 2015 S. Alkan and A. Secer. This is an open access article distributed under the Creative Commons Attribution License, which permits unrestricted use, distribution, and reproduction in any medium, provided the original work is properly cited.

We employ the sinc-Galerkin method to obtain approximate solutions of space-fractional order partial differential equations (FPDEs) with variable coefficients. The fractional derivatives are used in the Caputo sense. The method is applied to three different problems and the obtained solutions are compared with the exact solutions of the problems. These comparisons demonstrate that the sinc-Galerkin method is a very efficient tool in solving space-fractional partial differential equations.

\section{Introduction}

Fractional calculus, which might be considered as an extension of classical calculus, is as old as the classical calculus and fractional differential equations have been often used to describe many scientific phenomena in biomedical engineering, image processing, earthquake engineering, signal processing, physics, statistics, electrochemistry, and control theory.

Because finding the exact or analytical solutions of fractional order differential equations is not an easy task, several different numerical solution techniques have been developed for the approximate solutions of these types of equations. Some of the well-known numerical techniques might be listed as generalized differential transform method $[1,2]$, finite difference method [3], Adomian decomposition method [4, 5], homotopy perturbation method [6-8], Haar wavelet method $[9,10]$, differential transform method [11-13], and AdamsBashforth-Moulton scheme [14]. A detailed and informative study on fractional calculus can be found in [15]. Furthermore a relatively new analytical method was presented in [16] to solve time "The Time-Fractional Coupled-Korteweg-deVries Equations" via homotopy decomposition method by the same authors. The sinc methods were introduced in [17] and expanded in [18] by Frank Stenger. Sinc functions were firstly analyzed in $[19,20]$. In [21], the sinc-Galerkin method is used to approximate solutions of nonlinear differential equations with homogeneous and nonhomogeneous boundary conditions. In [22], the sinc-Galerkin method is applied to nonlinear fourth-order differential equations with nonhomogeneous and homogeneous boundary conditions. In the paper at [23], the numerical solutions of Troesch's problem are obtained by the sinc-Galerkin method and the results are compared with methods of Laplace, homotopy perturbation, splines, and perturbation. Reference [24] which contains short abstract version of current paper has been presented in an International Conference and Workshop on Mathematical Analysis 2014, Malaysia. In [25], the authors present a comparison between sinc-Galerkin method and sinc-collocation method to obtain approximate solutions of linear and nonlinear boundary value problems. Similarly, the wavelet-Galerkin method and the sinc-Galerkin method for solving nonhomogeneous heat equations are compared in [26]. The paper [27] offers an application of the sincGalerkin method for solving second-order singular Dirichlettype boundary value problems. In [28], the sinc-Galerkin method is used to approximate solutions of fractional order ordinary differential equations in Caputo sense.

In this paper we propose a new solution technique for approximate solution of space-fractional order partial 
differential equations (FPDEs) with variable coefficients and boundary conditions by using the sinc-Galerkin method that has almost not been employed for the space-fractional order partial differential equations in the form

$$
\begin{array}{r}
u_{t t}=a(x) u_{x x}+b(x){ }_{0}^{C} D_{x}^{\beta} u+c(x) u+f(x, t), \\
0<\beta<1
\end{array}
$$

with boundary conditions

$$
\begin{gathered}
u(0, t)=u(1, t)=0, \\
u(x, 0)=u(x, 1)=0,
\end{gathered}
$$

where ${ }_{0}^{C} D_{x}$ is Caputo fractional derivative operator.

The paper is organized as follows. Section 2 presents basic theorems of fractional calculus and sinc-Galerkin method. In Section 3, we use the sinc-Galerkin method to obtain an approximate solution of a general space-fractional partial differential equation. In Section 4, we present three examples in order to illustrate the effectiveness and accuracy of the present method. The obtained results are compared with the exact results.

\section{Preliminaries}

2.1. Fractional Calculus. In this section, we present the definitions of the fractional Riemann-Liouville derivative and the Caputo of fractional derivatives. By using these definitions, we give the definition of the integration by parts of fractional order.

Definition 1 (see [29]). Let $f:[a, b] \times[c, d] \rightarrow \mathbb{R}$ be a function; $\alpha$ is a positive real number, and $n$ is the integer. $\alpha, n$ satisfy the inequality $n-1 \leq \alpha<n$ and $\Gamma$ the Euler gamma function. Then,

(i) the left and right Riemann-Liouville fractional derivatives of order $\alpha$ with respect to $x$ of $f(x, t)$ function are given as

$$
\begin{aligned}
& { }_{a} D_{x}^{\alpha} f(x, t)=\frac{1}{\Gamma(n-\alpha)} \frac{d^{n}}{d x^{n}} \int_{a}^{x}(x-s)^{n-\alpha-1} f(s, t) d s, \\
& { }_{x} D_{b}^{\alpha} f(x, t)=\frac{(-1)^{n}}{\Gamma(n-\alpha)} \frac{d^{n}}{d x^{n}} \int_{x}^{b}(s-x)^{n-\alpha-1} f(s, t) d s,
\end{aligned}
$$

respectively;

(ii) the left and right Caputo fractional derivatives of order $\alpha$ with respect to $x$ of $f(x, t)$ function are given as

$$
\begin{gathered}
{ }_{a}^{C} D_{x}^{\alpha} f(x, t)=\frac{1}{\Gamma(n-\alpha)} \int_{a}^{x}(x-s)^{n-\alpha-1} \frac{\partial^{n} f(s, t)}{\partial s^{n}} d s, \\
{ }_{x}^{C} D_{b}^{\alpha} f(x, t)=\frac{1}{\Gamma(n-\alpha)} \int_{x}^{b}(-1)^{n}(s-x)^{n-\alpha-1} \frac{\partial^{n} f(s, t)}{\partial s^{n}} d s,
\end{gathered}
$$

Now, we can write the definition of integration by parts of fractional order by using the relations given in (3)-(6).

Definition 2. If $0<\alpha<1$ and $f$ is a function such that $f(a, t)=f(b, t)=0$, one can write

$$
\begin{aligned}
& \int_{a}^{b} g(x, t){ }_{a}^{C} D_{x}^{\alpha} f(x, t) d x=\int_{a}^{b} f(x, t){ }_{x} D_{b}^{\alpha} g(x, t) d x, \\
& \int_{a}^{b} g(x, t){ }_{x}^{C} D_{b}^{\alpha} f(x, t) d x=\int_{a}^{b} f(x, t){ }_{a} D_{x}^{\alpha} g(x, t) d x .
\end{aligned}
$$

2.2. Properties of Sinc Basis Functions and Quadrature Interpolations. In this section, we recall notations and definitions of the sinc function state some known results and derive some useful formulas to be used in the next sections of the present paper.

\subsubsection{The Sinc Basis Functions}

Definition 3 (see [30]). The function which defined all $z \in \mathbb{C}$ by

$$
\operatorname{sinc}(z)= \begin{cases}\frac{\sin (\pi z)}{\pi z}, & z \neq 0, \\ 1, & z=0\end{cases}
$$

is called the sinc function.

Definition 4 (see [30]). Let $f$ be a function defined on $\mathbb{R}$ and let $h>0$. Define the series

$$
C(f, h)(x)=\sum_{k=-\infty}^{\infty} f(k h) \operatorname{sinc}\left(\frac{x-k h}{h}\right),
$$

where from (8) we have

$$
\begin{aligned}
S(k, h)(x) & =\operatorname{sinc}\left(\frac{x-k h}{h}\right) \\
& = \begin{cases}\frac{\sin (\pi((x-k h) / h))}{\pi((x-k h) / h)} & x \neq k h, \\
1, & x=k h .\end{cases}
\end{aligned}
$$

If the series in (9) converges, it is called the Whittaker cardinal function of $f$. They are based on the infinite strip $D_{s}$ in the complex plane

$$
D_{s} \equiv\left\{w=u+i v:|v|<d \leq \frac{\pi}{2}\right\} .
$$

Generally, approximations can be constructed for infinite, semi-infinite, and finite intervals. Define the function

$$
w=\phi(z)=\ln \left(\frac{z}{1-z}\right)
$$

respectively. 


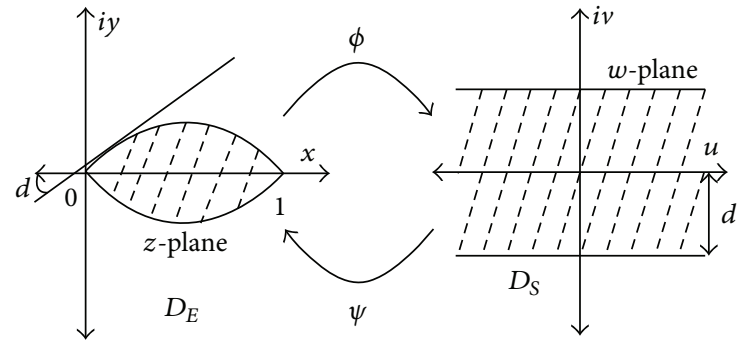

Figure 1: The domains $D_{E}$ and $D_{S}$.

which is a conformal mapping from $D_{E}$, the eye-shaped domain in the $z$-plane, onto the infinite strip $D_{S}$, where

$$
D_{E}=z=\left\{x+i y:\left|\arg \left(\frac{z}{1-z}\right)\right|<d \leq \frac{\pi}{2}\right\} .
$$

This is shown in Figure 1. For the sinc-Galerkin method, the bases functions are derived from the composite translated sinc functions

$$
S_{k}(z)=S(k, h)(z) \circ \phi(z)=\operatorname{sinc}\left(\frac{\phi(z)-k h}{h}\right),
$$

where $z \in D_{E}$. The function $z=\phi^{-1}(w)=e^{w} /\left(1+e^{w}\right)$ is an inverse mapping of $w=\phi(z)$. We may define the range of $\phi^{-1}$ on the real line as

$$
\Gamma=\left\{\phi^{-1}(u) \in D_{E}:-\infty<u<\infty\right\}
$$

evenly spaced nodes $\{k h\}_{k=-\infty}^{\infty}$ on the real line. The image which corresponds to these nodes is denoted by

$$
x_{k}=\phi^{-1}(k h)=\frac{e^{k h}}{1+e^{k h}} .
$$

\subsubsection{Sinc Function Interpolation and Quadrature}

Definition 5 (see [21]). Let $D_{E}$ be a simply connected domain in the complex plane $C$ and let $\partial D_{E}$ denote the boundary of $D_{E}$. Let $a, b$ be points on $\partial D_{E}$ and let $\phi$ be a conformal map $D_{E}$ onto $D_{S}$ such that $\phi(a)=-\infty$ and $\phi(b)=\infty$. If the inverse map of $\phi$ is denoted by $\varphi$, define

$$
\Gamma=\left\{\phi^{-1}(u) \in D_{E}:-\infty<u<\infty\right\},
$$

and $z_{k}=\varphi(k h), k= \pm 1, \pm 2, \ldots$

Definition 6 (see [21]). Let $B\left(D_{E}\right)$ be the class of functions $F$ that are analytic in $D_{E}$ and satisfy

$$
\int_{\psi(L+u)}|F(z)| d z \longrightarrow 0, \quad \text { as } u=\mp \infty
$$

in which

$$
L=\left\{i y:|y|<d \leq \frac{\pi}{2}\right\},
$$

and those on the boundary of $D_{E}$ satisfy

$$
T(F)=\int_{\partial D_{E}}|F(z) d z|<\infty
$$

Theorem 7 (see [21]). Let $\Gamma$ be $(0,1), F \in B\left(D_{E}\right)$; then, for $h>$ 0 sufficiently small,

$$
\begin{aligned}
& \int_{\Gamma} F(z) d z-h \sum_{j=-\infty}^{\infty} \frac{F\left(z_{j}\right)}{\phi^{\prime}\left(z_{j}\right)} \\
& \quad=\frac{i}{2} \int_{\partial D} \frac{F(z) k(\phi, h)(z)}{\sin (\pi \phi(z) / h)} d z \equiv I_{F},
\end{aligned}
$$

where

$$
|k(\phi, h)|_{z \in \partial D}=\left|e^{[(i \pi \phi(z) / h) \operatorname{sgn}(\operatorname{Im} \phi(z))]}\right|_{z \in \partial D}=e^{-\pi d / h} .
$$

For the sinc-Galerkin method, the infinite quadrature rule must be truncated to a finite sum. The following theorem indicates the conditions under which an exponential convergence results.

Theorem 8 (see [21]). If there exist positive constants $\alpha, \beta$, and $C$ such that

$$
\left|\frac{F(x)}{\phi^{\prime}(x)}\right| \leq C \begin{cases}e^{-\alpha|\phi(x)|}, & x \in \psi((-\infty, \infty)) \\ e^{-\beta|\phi(x)|}, & x \in \psi((0, \infty)),\end{cases}
$$

then the error bound for the quadrature rule (21) is

$$
\left|\int_{\Gamma} F(x) d x-h \sum_{j=-M}^{N} \frac{F\left(x_{j}\right)}{\phi^{\prime}\left(x_{j}\right)}\right| \leq C\left(\frac{e^{-\alpha M h}}{\alpha}+\frac{e^{-\beta N h}}{\beta}\right)+\left|I_{F}\right| .
$$

The infinite sum in (21) is truncated with the use of (23) to arrive at inequality (24). Making the selections

$$
\begin{gathered}
h=\sqrt{\frac{\pi d}{\alpha M},} \\
N \equiv \llbracket \frac{\alpha M}{\beta}+1 \rrbracket,
\end{gathered}
$$

where $\llbracket \cdot \rrbracket$ is an integer part of the statement and $M$ is the integer value which specifies the grid size, then

$$
\int_{\Gamma} F(x) d x=h \sum_{j=-M}^{N} \frac{F\left(x_{j}\right)}{\phi^{\prime}\left(x_{j}\right)}+O\left(e^{-(\pi \alpha d M)^{1 / 2}}\right) .
$$

We used these theorems to approximate the integrals that arise in the formulation of the discrete systems corresponding to a second-order boundary value problem.

\section{The Sinc-Galerkin Method}

Consider fractional boundary value problem

$$
u_{t t}=a(x) u_{x x}+b(x){ }_{0}^{C} D_{x}^{\beta} u+c(x) u+f(x, t), \quad 0<\beta<1
$$

with boundary conditions

$$
\begin{gathered}
u(0, t)=u(1, t)=0, \\
u(x, 0)=u(x, 1)=0,
\end{gathered}
$$


where ${ }_{0}^{C} D_{t}$ is Caputo fractional derivative operator. An approximate solution for $u(x, t)$ is represented by the formula

$$
u_{m_{x}, m_{t}}(x, t)=\sum_{j=-M_{t}}^{N_{t}} \sum_{i=-M_{x}}^{N_{x}} u_{i j} S_{i j}(x, t),
$$

where $m_{x}=M_{x}+N_{x}+1$ and $m_{t}=M_{t}+N_{t}+1$. The basis functions $\left\{S_{i j}(x, t)\right\}$ are given by

$$
S_{i j}(x, t)=S_{i}(x) S_{j}(t)=\left[S\left(i, h_{x}\right) \circ \phi(x)\right]\left[S\left(j, h_{t}\right) \circ \gamma(t)\right],
$$

where

$$
\begin{gathered}
\phi(x)=\ln \left(\frac{x}{1-x}\right), \\
\gamma(t)=\ln \left(\frac{t}{1-t}\right) .
\end{gathered}
$$

The unknown coefficients $u_{i j}$ in (29) are determined by orthogonalizing the residual with respect to the functions $\left\{S_{k l}(x, t)\right\},-M_{x} \leq k \leq N_{x},-M_{t} \leq l \leq N_{t}$. This yields the discrete Galerkin system

$$
\begin{array}{r}
\left\langle L u_{m_{x}, m_{t}}-f(x, t), S_{k l}\right\rangle=0, \\
-M_{x} \leq k \leq N_{x}, \quad-M_{t} \leq l \leq N_{t},
\end{array}
$$

where inner product is defined by

$$
\langle f, g\rangle=\iint_{0}^{1} f(x, t) g(x, t) W(x, t) d x d t,
$$

where $W(x)$ is weight function and it is convenient to take

$$
W(x, t)=w(x) v(t)=\left[\frac{1}{\left[\phi^{\prime}(x)\right]^{(1 / 2)}}\right]\left[\gamma^{\prime}(t)\right]^{-1 / 2}
$$

for the problem (27)-(28).

Lemma 9 (see [23]). Let $\phi$ be the conformal one-to-one mapping of the simply connected domain $D_{E}$ onto $D_{S}$, given by (12). Then

$$
\begin{gathered}
\delta_{j k}^{(0)}=\left.[S(j, h) \circ \phi(x)]\right|_{x=x_{k}}= \begin{cases}1, & j=k, \\
0, & j \neq k,\end{cases} \\
\delta_{j k}^{(1)}=\left.h \frac{d}{d \phi}[S(j, h) \circ \phi(x)]\right|_{x=x_{k}}= \begin{cases}0, & j=k, \\
\frac{(-1)^{k-j}}{k-j} & j \neq k,\end{cases} \\
\delta_{j k}^{(2)}=\left.h^{2} \frac{d^{2}}{d \phi^{2}}[S(j, h) \circ \phi(x)]\right|_{x=x_{k}}= \begin{cases}-\frac{\pi^{2}}{3}, & j=k, \\
\frac{-2(-1)^{k-j}}{(k-j)^{2}}, & j \neq k .\end{cases}
\end{gathered}
$$

The following theorems which can easily be proven by using Lemma 9 and definitions are used to solve (27).
Theorem 10 (see [31]). The following relations hold:

$$
\begin{gathered}
\left\langle u_{t t}, S_{k} S_{l}\right\rangle \cong h_{t} h_{x} \frac{w\left(x_{k}\right)}{\phi^{\prime}\left(x_{k}\right)} \sum_{j=-M_{t}}^{N_{t}} \sum_{i=0}^{2} \frac{u\left(x_{k}, t_{j}\right)}{\gamma^{\prime}\left(t_{j}\right)}\left[\frac{1}{h_{t}^{i}} \delta_{l j}^{(i)} \eta_{i}\right], \\
\left\langle a(x) u_{x x}, S_{k} S_{l}\right\rangle \cong h_{x} h_{t} \frac{v\left(t_{l}\right)}{\gamma^{\prime}\left(t_{l}\right)} \sum_{i=-M_{x}}^{N_{x}} \sum_{j=0}^{2} \frac{u\left(x_{i}, t_{l}\right)}{\phi^{\prime}\left(x_{i}\right)}\left[\frac{1}{h_{x}^{j}} \delta_{k i}^{(j)} \rho_{j}\right], \\
\left\langle c(x) u, S_{k} S_{l}\right\rangle \cong h_{t} h_{x} \frac{w\left(x_{k}\right) c\left(x_{k}\right) u\left(x_{k}, t_{l}\right) v\left(t_{l}\right)}{\phi^{\prime}\left(x_{k}\right) \gamma^{\prime}\left(t_{l}\right)}, \\
\left\langle f(x, t), S_{k} S_{l}\right\rangle \cong h_{t} h_{x} \frac{w\left(x_{k}\right) f\left(x_{k}, t_{l}\right) v\left(t_{l}\right)}{\phi^{\prime}\left(x_{k}\right) \gamma^{\prime}\left(t_{l}\right)},
\end{gathered}
$$

where

$$
\begin{gathered}
\eta_{2}=\left(\gamma^{\prime}\right)^{2} v, \\
\eta_{1}=\gamma^{\prime \prime} v+2 v^{\prime} \gamma^{\prime}, \\
\eta_{0}=v^{\prime \prime}, \\
\rho_{2}=\left(\phi^{\prime}\right)^{2} a w, \\
\rho_{1}=\phi^{\prime \prime} a w+2 \phi^{\prime}\left(a w^{\prime}+a^{\prime} w\right), \\
\rho_{0}=a^{\prime \prime} w+2 a^{\prime} w^{\prime}+a w^{\prime \prime} .
\end{gathered}
$$

Proof. See [31].

Theorem 11. For $0<\beta<1$, the following relations hold:

$$
\begin{aligned}
& \left\langle b(x){ }_{0}^{C} D_{x}^{\beta} u, S_{k} S_{l}\right\rangle \\
& \cong \frac{-h_{x} h_{t}}{\Gamma(1-\beta)} \frac{v\left(t_{l}\right)}{\gamma^{\prime}\left(t_{l}\right)} \\
& \quad \times\left.\sum_{i=-M_{x}}^{N_{x}} \frac{u\left(x_{i}, t_{l}\right)}{\phi^{\prime}\left(x_{i}\right)} \frac{d}{d x}\left[h_{P} \sum_{r=-P}^{P} \frac{\left(y_{r}-x\right)^{-\beta} R\left(y_{r}\right)}{\mu^{\prime}\left(y_{r}\right)}\right]\right|_{x=x_{i}},
\end{aligned}
$$

where $R(x)=b(x) S_{k}(x) w(x), \mu(s)=\ln ((s-x) /(1-s))$, and $h_{P}=\pi / \sqrt{P}$.

Proof. The inner product with sinc basis elements of $b(x){ }_{0}^{C} D_{x}^{\beta} u$ is given by

$$
\begin{aligned}
& \left\langle b(x){ }_{0}^{C} D_{x}^{\beta} u, S_{k} S_{l}\right\rangle \\
& \quad=\iint_{0}^{1} b(x){ }_{0}^{C} D_{x}^{\beta} u(x, t) S_{k}(x) S_{l}(t) w(x) v(t) d x d t .
\end{aligned}
$$


Using Definition 2, we can write

$$
\begin{aligned}
& \left\langle b(x){ }_{0}^{C} D_{x}^{\beta} u, S_{k} S_{l}\right\rangle \\
& =\iint_{0}^{1} b(x){ }_{0}^{C} D_{x}^{\beta} u(x, t) S_{k}(x) S_{l}(t) w(x) v(t) d x d t \\
& =\iint_{0}^{1} u(x, t) S_{l}(t) v(t){ }_{x} D_{1}^{\beta}\left(b(x) S_{k}(x) w(x)\right) d x d t,
\end{aligned}
$$

where $R(x)=b(x) S_{k}(x) w(x)$. By the definition of the Riemann-Liouville fractional derivative, we have

$$
{ }_{x} D_{1}^{\beta} R(x)=-\frac{1}{\Gamma(1-\beta)} \frac{d}{d x} \int_{x}^{1}(s-x)^{-\beta} R(s) d s .
$$

We will use the sinc quadrature rule given with (26) to compute it because the integral given in last equality is divergent on the interval $[t, 1]$. For this purpose, a conformal map and its inverse image that denotes the sinc grid points are given by

$$
\begin{gathered}
\mu(s)=\ln \left(\frac{s-x}{1-s}\right), \\
y_{r}=\mu^{-1}\left(r h_{P}\right)=\frac{e^{r h_{P}}+x}{1+e^{r h_{P}}},
\end{gathered}
$$

respectively. Then, according to equality (26) we can write

$$
\begin{aligned}
& -\frac{1}{\Gamma(1-\beta)} \frac{d}{d x} \int_{x}^{1}(s-x)^{-\beta} R(s) d s \\
& \cong-\frac{1}{\Gamma(1-\beta)} \frac{d}{d x}\left[h_{P} \sum_{r=-P}^{P} \frac{\left(y_{r}-x\right)^{-\beta} R\left(y_{r}\right)}{\mu^{\prime}\left(y_{r}\right)}\right]=N(x),
\end{aligned}
$$

where $h_{P}=\pi / \sqrt{P}$. As a result, it can be written in the following way:

$$
\left\langle b(x){ }_{0}^{C} D_{x}^{\beta} u, S_{k} S_{l}\right\rangle \cong \iint_{0}^{1} u(x, t) S_{l}(t) v(t) N(x) d x d t
$$

Now, applying the sinc quadrature rule with respect to $x$ and $t$ in last integral, we obtain

$$
\begin{aligned}
& \iint_{0}^{1} u(x, t) S_{l}(t) v(t) N(x) d x d t \\
& \quad \cong h_{x} h_{t} \sum_{i=M_{x}}^{N_{x}} \sum_{n=-M_{t}}^{N_{t}} \frac{u\left(x_{i}, t_{n}\right) v\left(t_{n}\right) S_{l}\left(t_{n}\right) N\left(x_{i}\right)}{\phi^{\prime}\left(x_{i}\right) \gamma^{\prime}\left(t_{n}\right)} .
\end{aligned}
$$

Consequently, using $\left.S_{l}(t)\right|_{t=t_{n}}=\delta_{\ln }^{(0)}=\delta_{\ln }$ we obtain

$$
\begin{aligned}
& \left\langle b(x){ }_{0}^{C} D_{x}^{\beta} u, S_{k} S_{l}\right\rangle \\
& \cong \frac{-h_{x} h_{t}}{\Gamma(1-\beta)} \frac{v\left(t_{l}\right)}{\gamma^{\prime}\left(t_{l}\right)} \\
& \quad \times\left.\sum_{i=-M_{x}}^{N_{x}} \frac{u\left(x_{i}, t_{l}\right)}{\phi^{\prime}\left(x_{i}\right)} \frac{d}{d x}\left[h_{P} \sum_{r=-P}^{P} \frac{\left(y_{r}-x\right)^{-\beta} R\left(y_{r}\right)}{\mu^{\prime}\left(y_{r}\right)}\right]\right|_{x=x_{i}} .
\end{aligned}
$$

This completes the proof.

Replacing each term of (32) with the approximation defined in Theorems 10 and 11 , replacing $u\left(x_{k}, t_{j}\right)$ by $u_{k j}$, and dividing by $h_{t} h_{x}$ we obtain the following theorem.

Theorem 12. If the assumed approximate solution of the boundary-value problem (27)-(28) is (29), then the discrete sinc-Galerkin system for the determination of the unknown coefficients $\left\{u_{k j},-M_{x} \leq k \leq N_{x},-M_{t} \leq j \leq N_{t}\right\}$ is given by

$$
\begin{aligned}
& \frac{w\left(x_{k}\right)}{\phi^{\prime}\left(x_{k}\right)} \sum_{j=-M_{t}}^{N_{t}} \sum_{i=0}^{2} \frac{u_{k j}}{\gamma^{\prime}\left(t_{j}\right)}\left[\frac{1}{h_{t}^{i}} \delta_{l j}^{(i)} \eta_{i}\right] \\
& =\frac{v\left(t_{l}\right)}{\gamma^{\prime}\left(t_{l}\right)} \sum_{i=-M_{x}}^{N_{x}} \sum_{j=0}^{2} \frac{u_{i l}}{\phi^{\prime}\left(x_{i}\right)}\left[\frac{1}{h_{x}^{j}} \delta_{k i}^{(j)} \rho_{j}\right] \\
& \quad-\frac{1}{\Gamma(1-\beta)} \frac{v\left(t_{l}\right)}{\gamma^{\prime}\left(t_{l}\right)} \\
& \quad \times\left.\sum_{i=-M_{x}}^{N_{x}} \frac{u_{i l}}{\phi^{\prime}\left(x_{i}\right)} \frac{d}{d x}\left[h_{P} \sum_{r=-P}^{P} \frac{\left(y_{r}-x\right)^{-\beta} R\left(y_{r}\right)}{\mu^{\prime}\left(y_{r}\right)}\right]\right|_{x=x_{i}} \\
& \quad+\frac{w\left(x_{k}\right) c\left(x_{k}\right) u_{k l} v\left(t_{l}\right)}{\phi^{\prime}\left(x_{k}\right) \gamma^{\prime}\left(t_{l}\right)} \\
& \quad+\frac{w\left(x_{k}\right) f\left(x_{k}, t_{l}\right) v\left(t_{l}\right)}{\phi^{\prime}\left(x_{k}\right) \gamma^{\prime}\left(t_{l}\right)} .
\end{aligned}
$$

We introduce the following notations in order the write the system above in a matrix-vector form. Let $I_{m_{x}}^{(P)}, P=0,1,2$ be the $m_{x} \times m_{x}$ matrices $I^{(P)}$, with $j k$ th entry $\delta_{j k}^{(P)}$ as given by Lemma 9. Further, $D\left(g_{x}\right)$ is an $m_{x} \times m_{x}$ diagonal matrix whose diagonal entries are

$$
\left[g\left(x_{-M_{x}}\right), g\left(x_{-M_{x}+1}\right), \ldots, g\left(x_{0}\right), \ldots, g\left(x_{N_{x}}\right)\right]^{T} .
$$


The matrices $I_{m_{t}}^{(P)}, P=0,1,2$, and $D\left(g_{t}\right)$ are similarly defined though of size $m_{t} \times m_{t}$. Introducing this notation in (47) leads to the matrix form

$$
\begin{aligned}
& D\left(\frac{1}{\phi^{\prime}}\right) D(w) U D(v)\left[\sum_{j=0}^{2} \frac{1}{h_{t}^{j}} I_{m_{t}}^{(j)} D\left(\frac{\eta_{j}}{\gamma^{\prime} v}\right)\right]^{t} \\
& -\left[\sum_{i=0}^{2} \frac{1}{h_{x}^{i}} I_{m_{x}}^{(i)} D\left(\frac{\rho_{i}}{\left(\phi^{\prime}\right)^{2} w}\right)\right] D\left(\phi^{\prime}\right) D(w) U D\left(\frac{v}{\gamma^{\prime}}\right) \\
& -\left[B D\left(\frac{1}{\left(\phi^{\prime}\right)^{2} w}\right)\right] D\left(\phi^{\prime}\right) D(w) U D\left(\frac{v}{\gamma^{\prime}}\right) \\
& -D\left(\frac{w}{\phi^{\prime}}\right) D(c) U D\left(\frac{v}{\gamma^{\prime}}\right)=D\left(\frac{w}{\phi^{\prime}}\right) F D\left(\frac{v}{\gamma^{\prime}}\right)
\end{aligned}
$$

where

$$
B=-\left.\frac{1}{\Gamma(1-\beta)} \frac{d}{d x}\left[h_{P} \sum_{r=-P}^{P} \frac{\left(y_{r}-x\right)^{-\beta} R\left(y_{r}\right)}{\mu^{\prime}\left(y_{r}\right)}\right]\right|_{x=x_{i}} .
$$

Firstly multiplying this term with $D\left(\phi^{\prime}\right)$ and secondly multiplying it with $D\left(\gamma^{\prime}\right)$ yield the equivalent system

$$
\Phi X+X \Psi=G
$$

where

$$
\begin{gathered}
\Phi=-A 1-A 2-C, \\
\Psi=B 1
\end{gathered}
$$

for

$$
\begin{gathered}
A 1=D\left(\phi^{\prime}\right)\left[\sum_{i=0}^{2} \frac{1}{h_{x}^{i}} I_{m_{x}}^{(i)} D\left(\frac{\rho_{i}}{\left(\phi^{\prime}\right)^{2} w}\right)\right] D\left(\phi^{\prime}\right), \\
A 2=D\left(\phi^{\prime}\right)\left[B D\left(\frac{1}{\left(\phi^{\prime}\right)^{2} w}\right)\right] D\left(\phi^{\prime}\right), \\
B 1=\left[\sum_{j=0}^{2} \frac{1}{h_{t}^{j}} I_{m_{t}}^{(j)} D\left(\frac{\eta_{j}}{\gamma^{\prime} v}\right)\right]^{t} D\left(\gamma^{\prime}\right), \\
C=D(c) .
\end{gathered}
$$

Furthermore,

$$
\begin{aligned}
& G=D(w) F D(v), \\
& X=D(w) U D(v) .
\end{aligned}
$$

$\Phi, \Psi, X$, and $G$ have dimensions $m_{x} \times m_{x}, m_{t} \times m_{t}, m_{x} \times m_{t}$, and $m_{x} \times m_{t}$, respectively. At last, the $m_{x} \times m_{t}$ matrices $U$ and $F$ have klth entries given by $u_{k l}$ and $f\left(x_{k}, t_{l}\right)=f\left(e^{k h} /(1+\right.$ $\left.\left.e^{k h}\right), e^{l h} /\left(1+e^{l h}\right)\right)$, respectively.

To obtain the approximate solution equation (29), we need to solve the system for $U$ which requires solving (51) for $X$. Solution of (51) for $X$ is shown in [26].

\section{Examples}

In this section, the present method will be tested on three different problems.

Example 1. Consider fractional boundary value problem

$$
\begin{gathered}
u_{t t}=u_{x x}+{ }_{0}^{C} D_{x}^{0.7} u+f(x, t), \\
u(0, t)=u(1, t)=0, \\
u(x, 0)=u(x, 1)=0
\end{gathered}
$$

which has the following exact solution:

$$
u(x, t)=x^{2}(1-x) t^{3}(1-t)^{2}
$$

for

$$
\begin{aligned}
f(x, t)= & -2(1-t)^{2} t^{3}(1-x)+4(1-t)^{2} t^{3} x \\
& +6(1-t)^{2} t(1-x) x^{2}-12(1-t) t^{2} \\
& \times(1-x) x^{2}+2 t^{3}(1-x) x^{2}+0.334273 \\
& \times(-1+t)^{2} t^{3} x^{1.3}(-5.12821+6.68896 x) .
\end{aligned}
$$

The numerical solutions which are obtained by using the sincGalerkin method (SGM) for this problem are presented in Tables 1 and 2 for different values. Also, the graphs of exact and approximate solutions for different values are presented in Figures 2 and 3.

Example 2. Consider fractional boundary value problem

$$
\begin{gathered}
u_{t t}=e^{x} u_{x x}+\left(x^{2}+1\right){ }_{0}^{C} D_{x}^{0.3} u-\frac{1}{x+1} u+f(x, t) \\
u(0, t)=u(1, t)=0 \\
u(x, 0)=u(x, 1)=0
\end{gathered}
$$

which has the following exact solution:

$$
u(x, t)=x^{2}(1-x) \sin (\pi t)
$$

for

$$
f(x, t)
$$

$$
\begin{aligned}
= & -\pi^{2}(1-x) x^{2} \sin (\pi t)+\frac{(1-x) x^{2} \sin (\pi t)}{1+x} \\
& -0.770383\left(1+x^{2}\right)\left(1.68067 x^{1.7}-1.86741 x^{2.7}\right) \\
& \times \sin (\pi t)-e^{x}(2(1-x) \sin (\pi t)-4 x \sin (\pi t)) .
\end{aligned}
$$

The numerical solutions which are obtained by using the sincGalerkin method (SGM) for this problem are presented in Tables 3 and 4. In addition, in Figures 4 and 5, the graphs of exact and approximate solutions for different values are presented. 


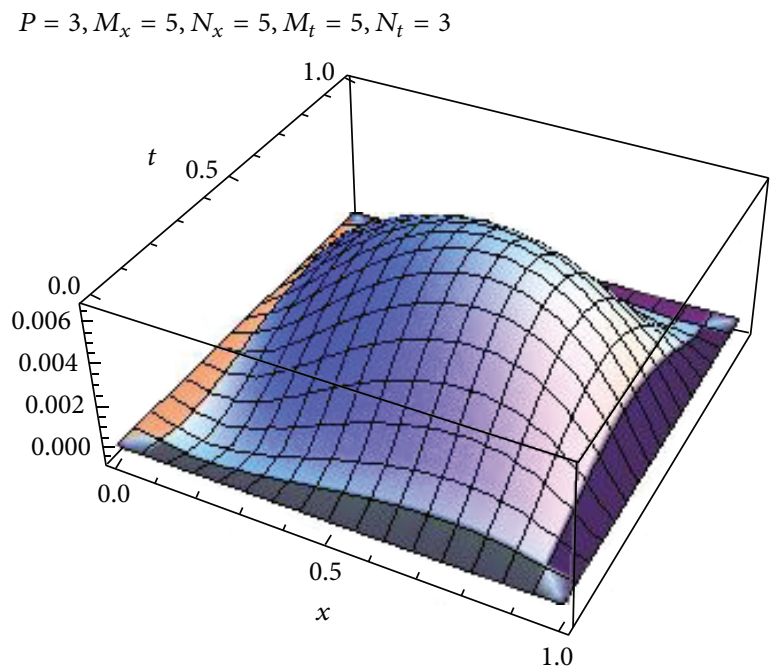

$$
P=20, M_{x}=40, N_{x}=40, M_{t}=40, N_{t}=30
$$

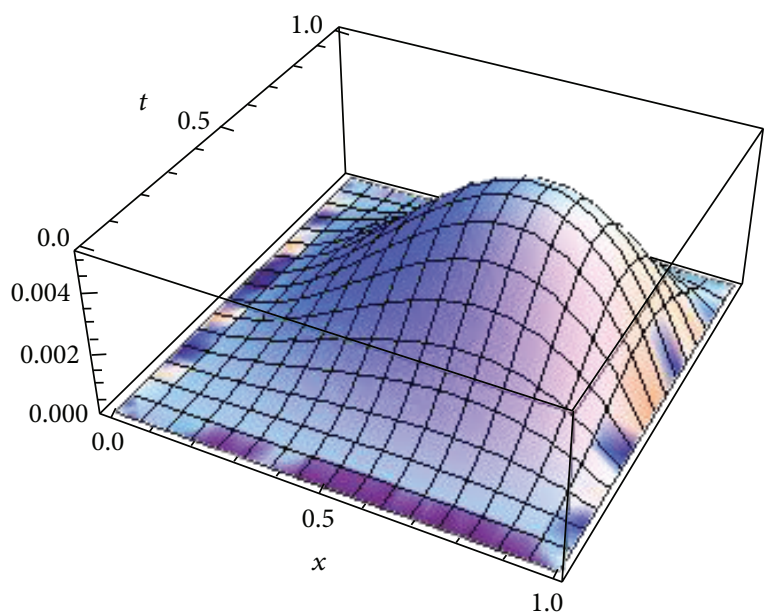

FIGURE 2: Graphs of approximate solutions for different values.

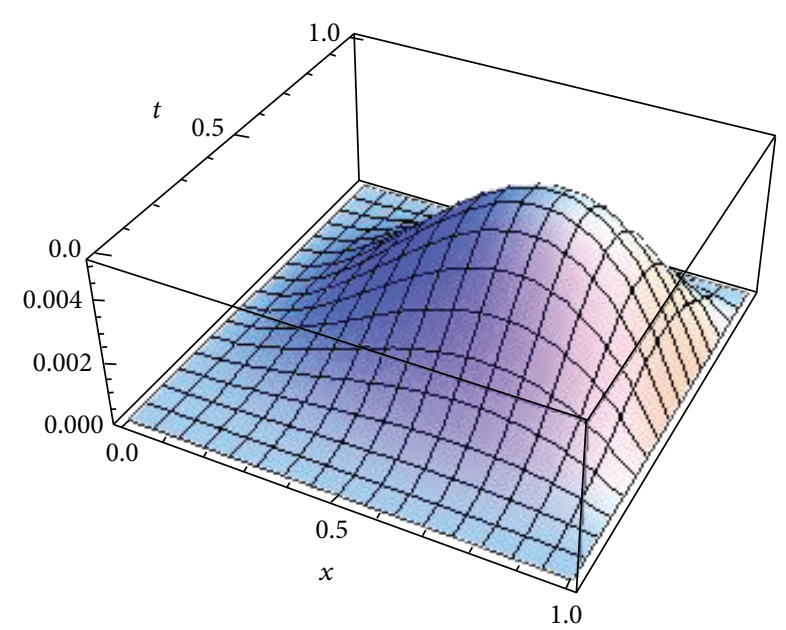

FIGURE 3: Graph of exact solution.

Example 3 (see [32]). Consider the fractional convection-diffusion equation

$$
\begin{gathered}
\frac{\partial^{\gamma} u(x, t)}{\partial t^{\gamma}}=-a(x) \frac{\partial^{\alpha} u(x, t)}{\partial x^{\alpha}}+b(x) \frac{\partial^{\beta} u(x, t)}{\partial x^{\beta}}+f(x, t) \\
0<\gamma \leq 2, \quad 1<\alpha \leq 2, \quad 0<\beta \leq 1, \\
u(0, t)=u(1, t)=0, \\
u(x, 0)=u(x, 1)=0 .
\end{gathered}
$$

In particular, if $\gamma=2, \alpha=2, \beta=0.35, a(x)=\Gamma(2.35) \Gamma(2.65)$ $x^{0.35}, b(x)=\Gamma(0.7) \Gamma(1) x^{2}$.

The problem has the following exact solution:

$$
u(x, t)=\left(x^{1.7}-x^{2}\right) \sin (2 \pi t)
$$

TABle 1: Numerical results for $P=3, M_{x}=5, N_{x}=5, M_{t}=5$, $N_{t}=3$.

\begin{tabular}{lcccc}
\hline$t$ & $x$ & Exact sol. & Num. sol. & Error \\
\hline \multirow{3}{*}{0.03} & 0.3 & 0.00000160 & 0.000069824 & 0.000068223 \\
& 0.6 & 0.00000365 & 0.000557026 & 0.000553368 \\
& 0.9 & 0.00000205 & 0.000286490 & 0.000284433 \\
\hline \multirow{3}{*}{0.06} & 0.3 & 0.00001202 & -0.000160475 & 0.000172499 \\
& 0.6 & 0.00002748 & 0.000688434 & 0.000660950 \\
& 0.9 & 0.00001545 & 0.000405155 & 0.000389695 \\
\hline \multirow{3}{*}{0.09} & 0.3 & 0.00003803 & -0.000317899 & 0.000355931 \\
& 0.6 & 0.00008693 & 0.000879725 & 0.000792795 \\
& 0.9 & 0.00004889 & 0.000577303 & 0.000528404 \\
\hline
\end{tabular}

TABLE 2: Numerical results for $P=20, M_{x}=40, N_{x}=40, M_{t}=40$, $N_{t}=30$.

\begin{tabular}{lcccc}
\hline$t$ & $x$ & Exact sol. & Num. sol. & Error \\
\hline \multirow{3}{*}{0.03} & 0.3 & 0.00000160 & 0.00000376 & $2.16753 * 10^{-6}$ \\
& 0.6 & 0.00000365 & 0.00001237 & $8.71244 * 10^{-6}$ \\
& 0.9 & 0.00000205 & 0.00000667 & $4.61338 * 10^{-6}$ \\
\hline \multirow{2}{*}{0.06} & 0.3 & 0.00001202 & 0.00001645 & $4.43271 * 10^{-6}$ \\
& 0.6 & 0.00002748 & 0.00004476 & $1.72861 * 10^{-5}$ \\
& 0.9 & 0.00001545 & 0.00002455 & $9.09322 * 10^{-6}$ \\
\hline \multirow{3}{*}{0.09} & 0.3 & 0.00003803 & 0.00004497 & $6.93846 * 10^{-6}$ \\
& 0.6 & 0.00008693 & 0.00011262 & $2.56904 * 10^{-5}$ \\
& 0.9 & 0.00004889 & 0.00006228 & $1.33882 * 10^{-5}$ \\
\hline
\end{tabular}

for

$$
\begin{aligned}
f(x, t)= & \left(2 \pi x^{1.7}-x^{2}\right) t^{-1} E_{2,0}\left(-(2 \pi t)^{2}\right) \\
& +\left\{\Gamma(2.7)(\Gamma(2.65)-\Gamma(1)) x^{1.7}\right. \\
& \left.+\Gamma(3)(\Gamma(0.7)-\Gamma(2.35)) x^{2}\right\} \sin (2 \pi t) .
\end{aligned}
$$



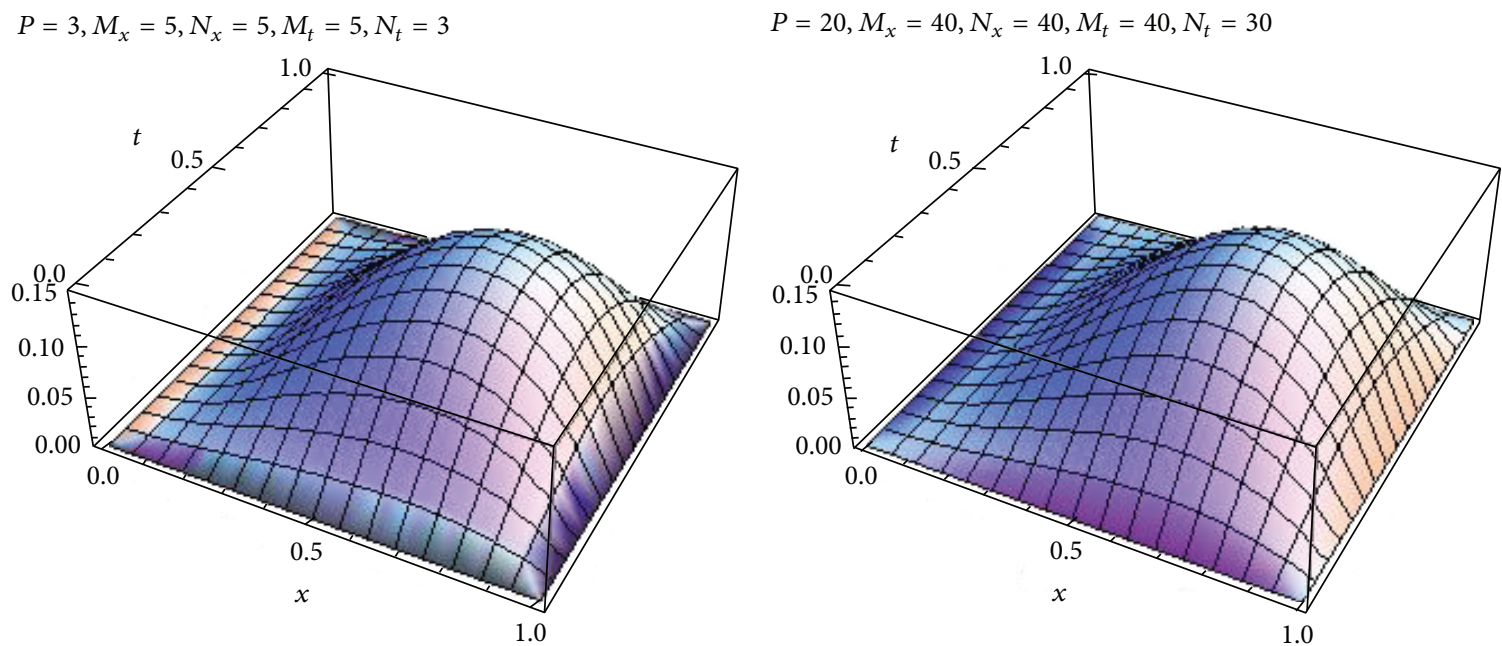

FIGURE 4: Graphs of approximate solutions for different values.

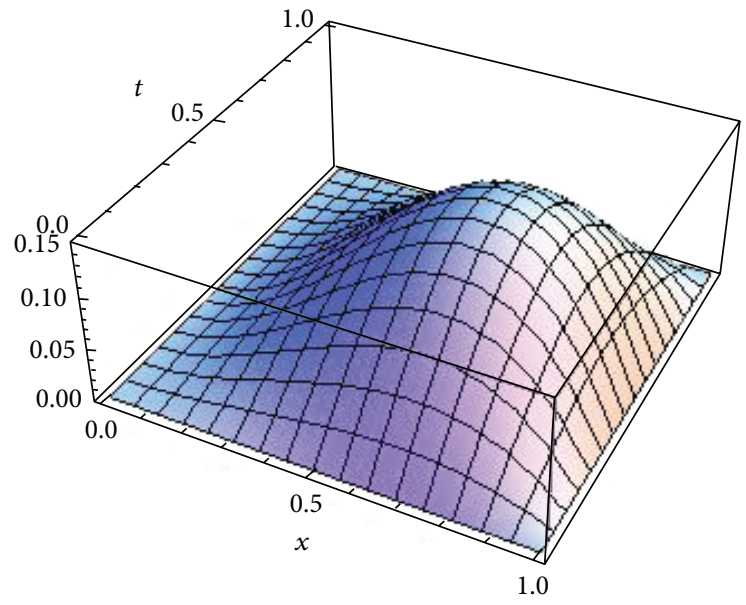

FIGURE 5: Graphs of exact solution.

TABLe 3: Numerical results for $P=3, M_{x}=5, N_{x}=5, M_{t}=5$, $N_{t}=3$.

\begin{tabular}{lcccc}
\hline$t$ & $x$ & Exact sol. & Num. sol. & Error \\
\hline \multirow{4}{*}{0.03} & 0.3 & 0.0059288 & 0.0071562 & 0.00122741 \\
& 0.6 & 0.0135516 & 0.0149881 & 0.00143653 \\
& 0.9 & 0.0076227 & 0.0083725 & 0.00074978 \\
\hline \multirow{2}{*}{0.06} & 0.3 & 0.0118050 & 0.0131991 & 0.00139409 \\
& 0.6 & 0.0269829 & 0.0254147 & 0.00156823 \\
& 0.9 & 0.0151779 & 0.0145261 & 0.00065176 \\
\hline \multirow{2}{*}{0.09} & 0.3 & 0.0175764 & 0.0193179 & 0.00174151 \\
& 0.6 & 0.0401747 & 0.0362484 & 0.00392631 \\
& 0.9 & 0.0225983 & 0.0208906 & 0.00170767 \\
\hline
\end{tabular}

The numerical solutions which are obtained by using the sincGalerkin method (SGM) for this problem are presented in Tables 5 and 6. In addition, in Figures 6 and 7, the graphs of exact and approximate solutions for different values are presented.
TABLE 4: Numerical results for $P=20, M_{x}=40, N_{x}=40, M_{t}=40$, $N_{t}=30$.

\begin{tabular}{lcccc}
\hline$t$ & $x$ & Exact sol. & Num. sol. & Error \\
\hline \multirow{4}{*}{0.03} & 0.3 & 0.0059288 & 0.0059287 & $1.16151 * 10^{-7}$ \\
& 0.6 & 0.0135516 & 0.0135515 & $1.41292 * 10^{-7}$ \\
& 0.9 & 0.0076227 & 0.0076227 & $1.58382 * 10^{-8}$ \\
\hline \multirow{3}{*}{0.06} & 0.3 & 0.0118050 & 0.0118048 & $2.68162 * 10^{-7}$ \\
& 0.6 & 0.0269829 & 0.0269826 & $3.57381 * 10^{-7}$ \\
& 0.9 & 0.0151779 & 0.0151778 & $7.75388 * 10^{-8}$ \\
\hline \multirow{3}{*}{0.09} & 0.3 & 0.0175764 & 0.0175761 & $3.24333 * 10^{-7}$ \\
& 0.6 & 0.0401747 & 0.0401744 & $3.45727 * 10^{-7}$ \\
& 0.9 & 0.0225983 & 0.0225983 & $1.68470 * 10^{-8}$ \\
\hline
\end{tabular}

TAble 5: Numerical results for $P=3, M_{x}=5, N_{x}=5, M_{t}=5$, $N_{t}=3$.

\begin{tabular}{lcccc}
\hline$t$ & $x$ & Exact sol. & Num. sol. & Error \\
\hline \multirow{4}{*}{0.03} & 0.3 & 0.0073366 & 0.0043780 & 0.00295855 \\
& 0.6 & 0.0111718 & 0.0092590 & 0.00191284 \\
& 0.9 & 0.0048740 & 0.0045355 & 0.00033852 \\
\hline \multirow{2}{*}{0.06} & 0.3 & 0.0144134 & 0.0107858 & 0.00362752 \\
& 0.6 & 0.0219479 & 0.0228301 & 0.00088218 \\
& 0.9 & 0.0095754 & 0.0110942 & 0.00151873 \\
\hline \multirow{3}{*}{0.09} & 0.3 & 0.0209795 & 0.0167664 & 0.00421307 \\
& 0.6 & 0.0319465 & 0.0354700 & 0.00352348 \\
& 0.9 & 0.0139377 & 0.0172001 & 0.00326239 \\
\hline
\end{tabular}

\section{Conclusion}

In this study, we use the sinc-Galerkin method to obtain approximate solutions of boundary value problems for spacefractional partial differential equations with variable coefficients. In order to illustrate the efficiency and accuracy of the present method, the method is applied to three examples in the literature and the obtained results are compared with exact solutions. As a result, it is shown that sinc-Galerkin 

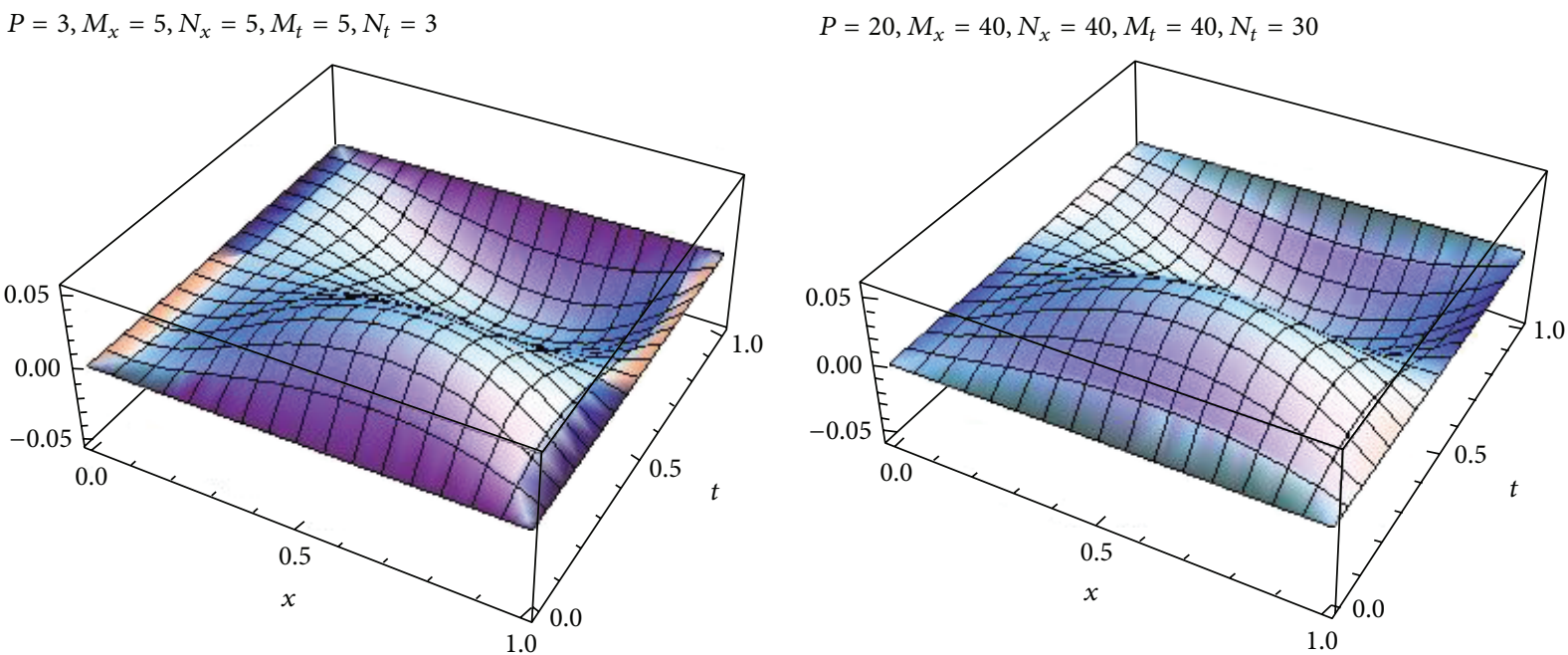

FIGURE 6: Graphs of approximate solutions for different values.

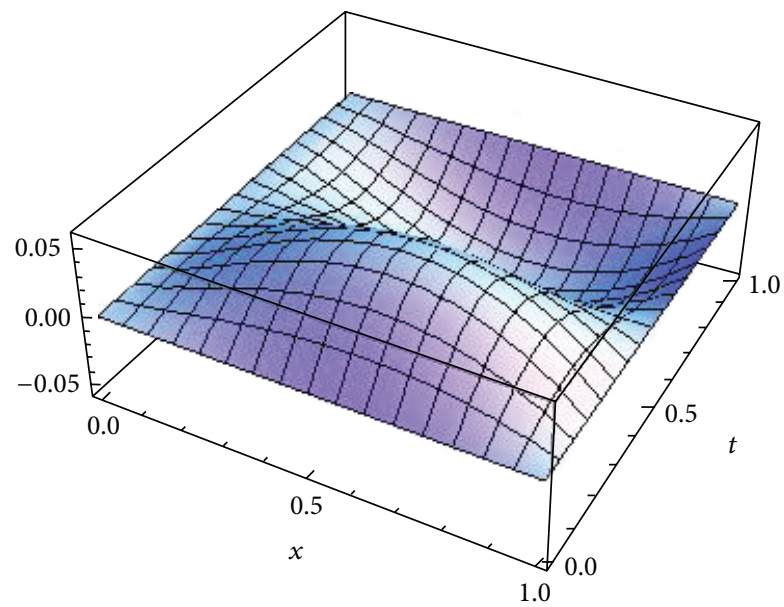

FIgURE 7: Graphs of exact solution.

TABLE 6: Numerical results for $P=20, M_{x}=40, N_{x}=40, M_{t}=$ $40, N_{t}=30$.

\begin{tabular}{lcccc}
\hline$t$ & $x$ & Exact sol. & Num. sol. & Error \\
\hline \multirow{4}{*}{0.03} & 0.3 & 0.0073366 & 0.0073365 & $6.80615 * 10^{-8}$ \\
& 0.6 & 0.0111718 & 0.0111718 & $2.45596 * 10^{-8}$ \\
& 0.9 & 0.0048740 & 0.0048740 & $1.74662 * 10^{-8}$ \\
\hline \multirow{2}{*}{0.06} & 0.3 & 0.0144134 & 0.0144139 & $5.33686 * 10^{-7}$ \\
& 0.6 & 0.0219479 & 0.0219489 & $9.67538 * 10^{-7}$ \\
& 0.9 & 0.0095754 & 0.0095759 & $4.80610 * 10^{-7}$ \\
\hline \multirow{3}{*}{0.09} & 0.3 & 0.0209795 & 0.0209786 & $8.62732 * 10^{-7}$ \\
& 0.6 & 0.0319465 & 0.0319454 & $1.08969 * 10^{-6}$ \\
& 0.9 & 0.0139377 & 0.0139373 & $3.85036 * 10^{-7}$ \\
\hline
\end{tabular}

method is very effective and accurate for obtaining approximate solutions of space-fractional differential equations with variable coefficients. In the future, we plan to extend the present numerical solution technique to nonlinear spacefractional partial differential equations.

\section{Conflict of Interests}

The authors declare that there is no conflict of interests regarding the publication of this paper.

\section{References}

[1] S. Momani, Z. Odibat, and V. S. Erturk, "Generalized differential transform method for solving a space- and time-fractional diffusion-wave equation," Physics Letters A, vol. 370, no. 5-6, pp. 379-387, 2007.

[2] Z. Odibat and S. Momani, "A generalized differential transform method for linear partial differential equations of fractional order," Applied Mathematics Letters, vol. 21, no. 2, pp. 194-199, 2008.

[3] Y. Zhang, "A finite difference method for fractional partial differential equation," Applied Mathematics and Computation, vol. 215, no. 2, pp. 524-529, 2009.

[4] Q. Wang, "Numerical solutions for fractional KdV-Burgers equation by Adomian decomposition method," Applied Mathematics and Computation, vol. 182, no. 2, pp. 1048-1055, 2006.

[5] V. Daftardar-Gejji and S. Bhalekar, "Solving multi-term linear and non-linear diffusion-wave equations of fractional order by Adomian decomposition method," Applied Mathematics and Computation, vol. 202, no. 1, pp. 113-120, 2008.

[6] Q. Wang, "Homotopy perturbation method for fractional KdV equation," Applied Mathematics and Computation, vol. 190, no. 2, pp. 1795-1802, 2007.

[7] Q. Wang, "Homotopy perturbation method for fractional KdVBurgers equation," Chaos, Solitons and Fractals, vol. 35, no. 5, pp. 843-850, 2008.

[8] O. Abdulaziz, I. Hashim, and E. S. Ismail, "Approximate analytical solution to fractional modified KdV equations," Mathematical and Computer Modelling, vol. 49, no. 1-2, pp. 136-145, 2009.

[9] M. U. Rehman and R. A. Khan, "Numerical solutions to initial and boundary value problems for linear fractional partial differential equations," Applied Mathematical Modelling, vol. 37, no. 7, pp. 5233-5244, 2013. 
[10] M. A. Akinlar, A. Secer, and M. Bayram, "Numerical solution of fractional Benney equation," Applied Mathematics and Information Sciences, vol. 8, no. 4, pp. 1633-1637, 2014.

[11] A. Secer, M. A. Akinlar, and A. Cevikel, "Efficient solutions of systems of fractional PDEs by the differential transform method," Advances in Difference Equations, vol. 2012, article 188, 7 pages, 2012.

[12] M. Kurulay and M. Bayram, "Approximate analytical solution for the fractional modified $\mathrm{KdV}$ by differential transform method," Communications in Nonlinear Science and Numerical Simulation, vol. 15, no. 7, pp. 1777-1782, 2010.

[13] M. Kurulay, M. A. Akinlar, and R. Ibragimov, "Computational solution of a fractional integro-differential equation," Abstract and Applied Analysis, vol. 2013, Article ID 865952, 4 pages, 2013.

[14] M. A. Akinlar, A. Secer, and M. Bayram, "Stability, synchronization control and numerical solution of fractional ShimizuMorioka dynamical system," Applied Mathematics \& Information Sciences, vol. 8, no. 4, pp. 1699-1705, 2014.

[15] A. Atangana and A. Secer, "A note on fractional order derivatives and table of fractional derivatives of some special functions," Abstract and Applied Analysis, vol. 2013, Article ID 279681, 8 pages, 2013.

[16] A. Atangana and A. Secer, "The time-fractional coupled-Korteweg-de-Vries equations," Abstract and Applied Analysis, vol. 2013, Article ID 947986, 8 pages, 2013.

[17] F. Stenger, "Approximations via Whittaker's cardinal function," Journal of Approximation Theory, vol. 17, no. 3, pp. 222-240, 1976.

[18] F. Stenger, "A sinc-Galerkin method of solution of boundary value problems," Mathematics of Computation, vol. 33, no. 145, pp. 85-109, 1979.

[19] E. T. Whittaker, "On the functions which are represented by the expansions of the interpolation theory," Proceedings of the Royal Society of Edinburgh, vol. 35, pp. 181-194, 1915.

[20] J. M. Whittaker, Interpolation Function Theory, vol. 33 of Cambridge Tracts in Mathematics and Mathematical Physics, Cambridge University Press, London, UK, 1935.

[21] M. El-Gamel and A. I. Zayed, "Sinc-Galerkin method for solving nonlinear boundary-value problems," Computers \& Mathematics with Applications, vol. 48, no. 9, pp. 1285-1298, 2004.

[22] M. El-Gamel, S. H. Behiry, and H. Hashish, "Numerical method for the solution of special nonlinear fourth-order boundary value problems," Applied Mathematics and Computation, vol. 145, no. 2-3, pp. 717-734, 2003.

[23] M. Zarebnia and M. Sajjadian, "The sinc-Galerkin method for solving Troesch's problem," Mathematical and Computer Modelling, vol. 56, no. 9-10, pp. 218-228, 2012.

[24] A. Seçer, S. Alkan, and M. Bayram, "Sinc-galerkin method for approximate solutions of space fractional partial differential equations," in Proceedings of the International Conference and Workshop on Mathematical Analysis (ICWOMA '14), p. 48, 2014.

[25] A. Mohsen and M. El-Gamel, "On the Galerkin and collocation methods for two-point boundary value problems using sinc bases," Computers \& Mathematics with Applications, vol. 56, no. 4, pp. 930-941, 2008.

[26] M. El-Gamel and A. I. Zayed, "A comparison between the wavelet-GALerkin and the sinc-GALerkin methods in solving nonhomogeneous heat equations," in Inverse Problem, Image Analysis, and Medical Imaging, vol. 313, pp. 97-116, 2002.
[27] A. Secer and M. Kurulay, "The sinc-Galerkin method and its applications on singular Dirichlet-type boundary value problems," Boundary Value Problems, vol. 2012, article 126, 14 pages, 2012.

[28] A. Secer, S. Alkan, M. A. Akinlar, and M. Bayram, "SincGalerkin method for approximate solutions of fractional order boundary value problems," Boundary Value Problems, vol. 2013, article 281, 2013.

[29] R. Almeida and D. F. M. Torres, "Necessary and sufficient conditions for the fractional calculus of variations with Caputo derivatives," Communications in Nonlinear Science and Numerical Simulation, vol. 16, no. 3, pp. 1490-1500, 2011.

[30] J. Lund and K. L. Bowers, Sinc Methods for Quadrature and Differential Equations, SIAM, Philadelphia, 1992.

[31] M. El-Gamel, "A numerical scheme for solving nonhomogeneous time-dependent problems," Zeitschrift für Angewandte Mathematik und Physik, vol. 57, no. 3, pp. 369-383, 2006.

[32] M. ur Rehman and R. A. Khan, "Numerical solutions to initial and boundary value problems for linear fractional partial differential equations," Applied Mathematical Modelling, vol. 37, no. 7, pp. 5233-5244, 2013. 


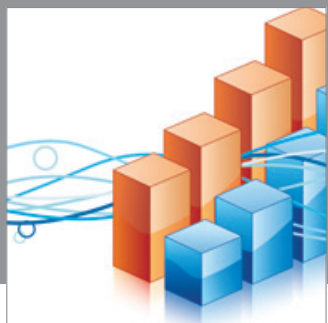

Advances in

Operations Research

mansans

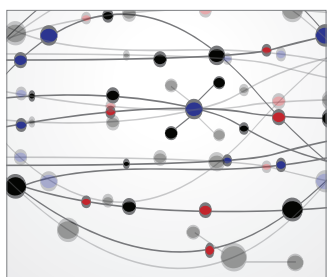

The Scientific World Journal
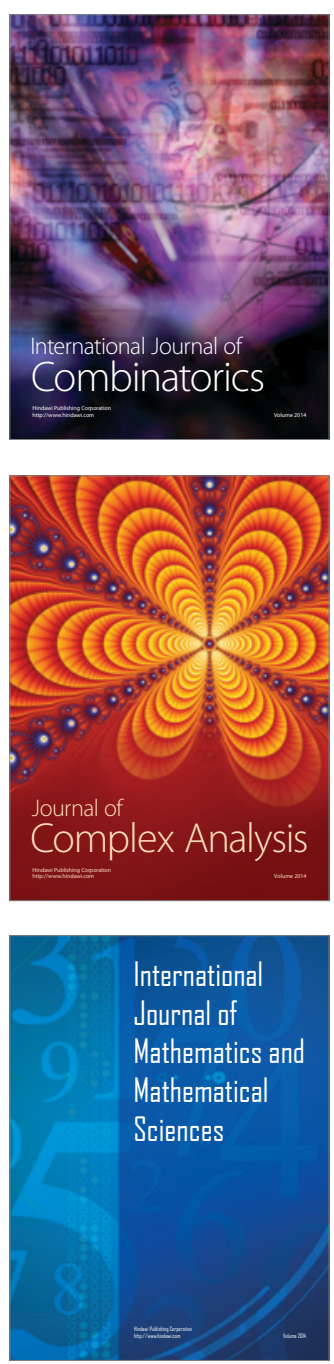
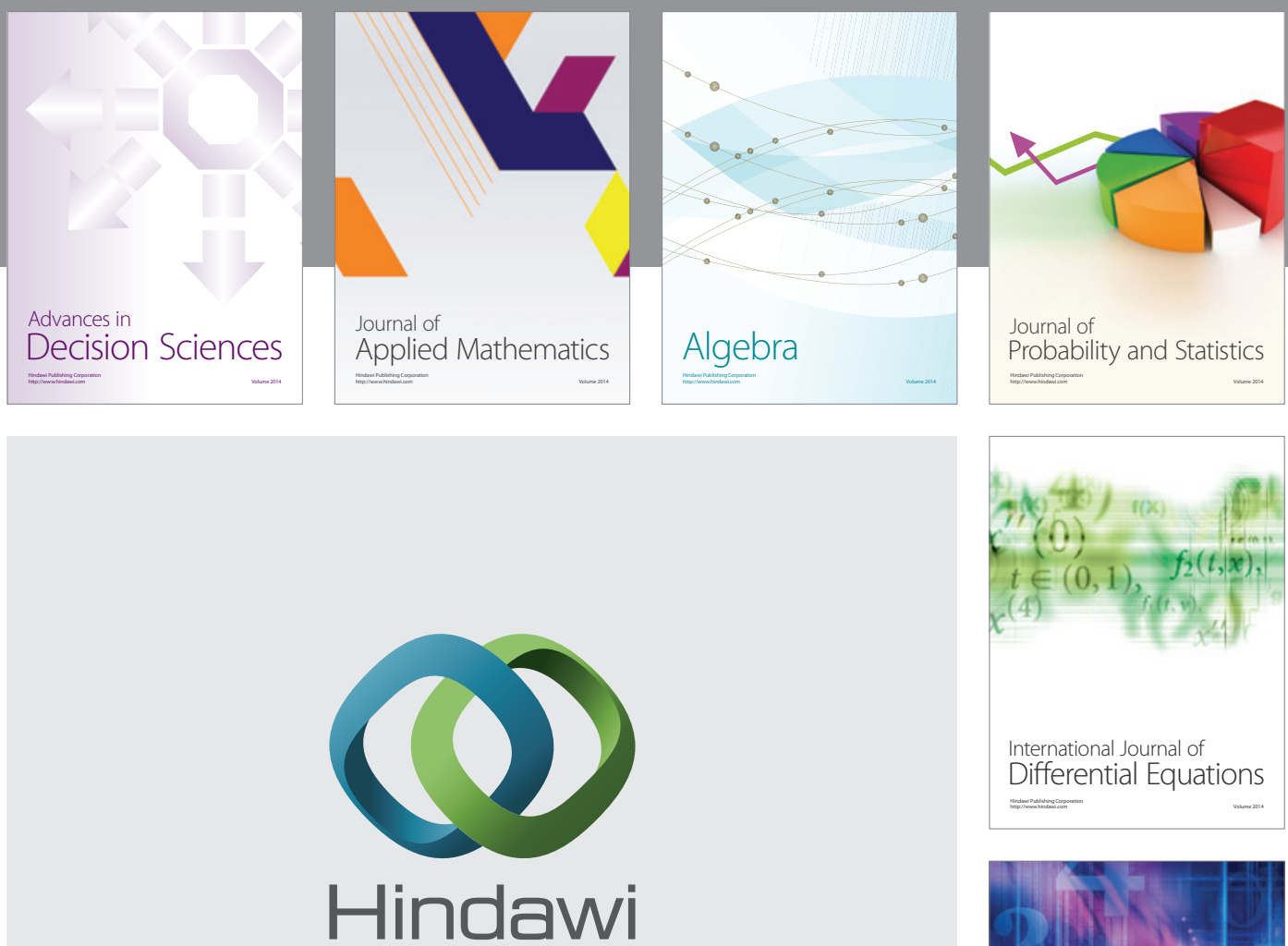

Submit your manuscripts at http://www.hindawi.com
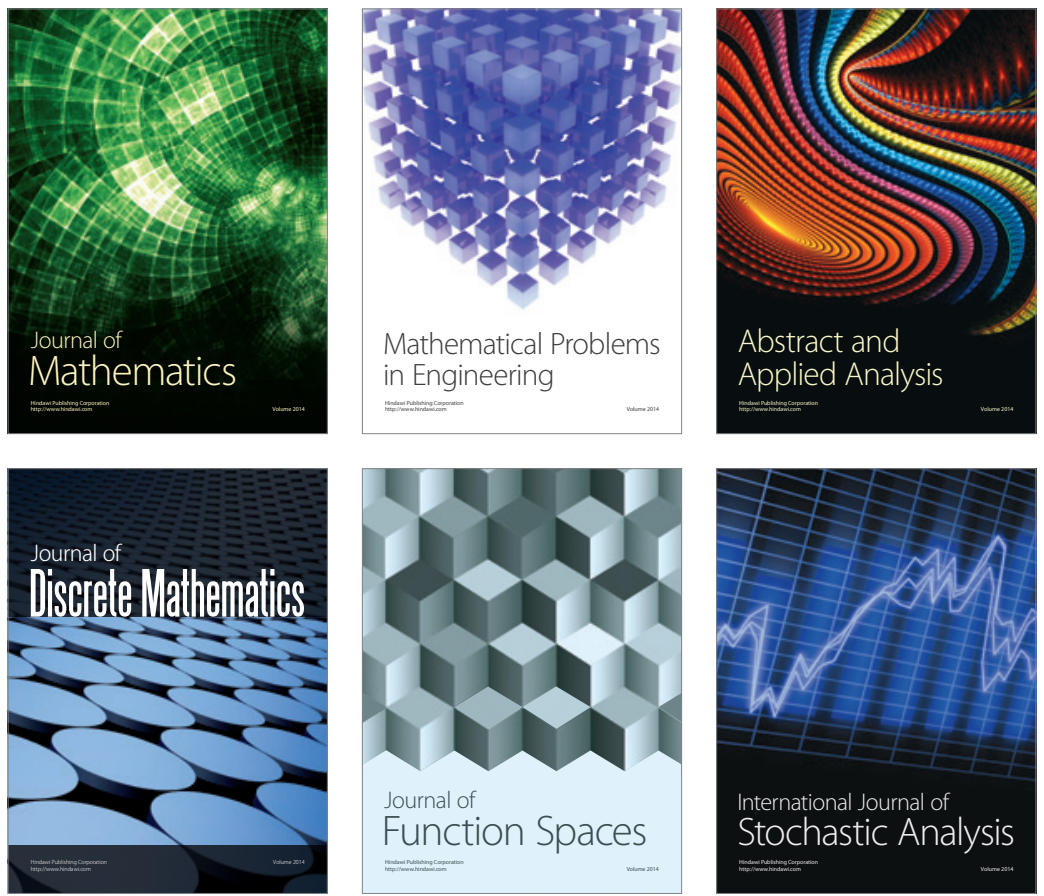

Journal of

Function Spaces

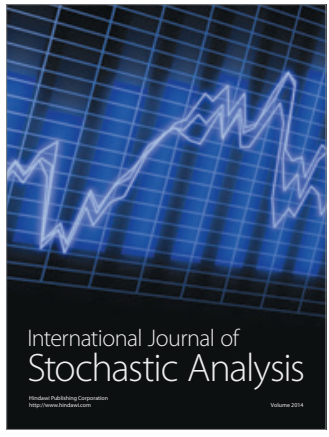

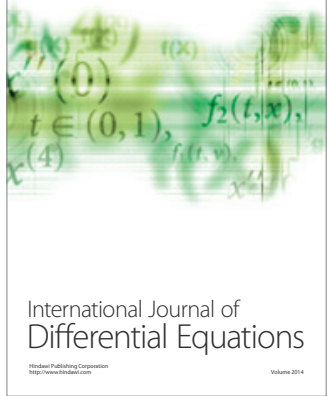
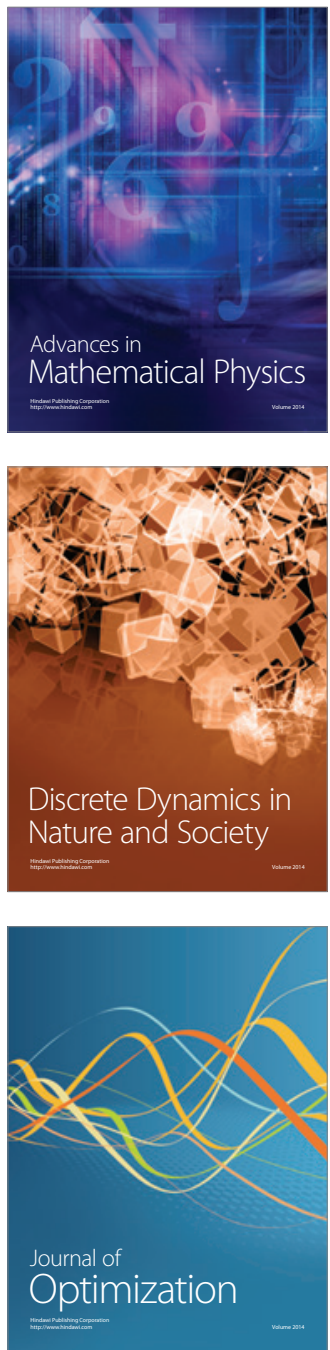